\title{
ALTERAÇÕES ELETRENCEFALOGRÁFRICAS NA SÍNDROME DE DOWN
}

\author{
ANA MOEMA PEREIRA DA NÓBREGA*, HENRIQUE GIL DA SILVA NUNESMAIA**, \\ NANCY DE OLIVEIRA VIANA***, MARCOS AURÉLIO SMITH FILGUEIRAS****
}

\begin{abstract}
RESUMO - A frequência da epilepsia na síndrome de Down (SD) tem sido registrada na literatura variando de 6 a 17\%. Um padrão eletrencefalográfico próprio da SD não foi determinado, existindo grande variação nas alterações eletrencefalográficas encontradas, não estando estas bem correlacionadas com alterações comportamentais ou sinais neurológicos. O principal objetivo deste estudo foi identificar parâmetros epidemiológicos e eletrencefalográficos acompanhados ou não de crises epilépticas em pacientes institucionalizados com diagnóstico clínico de SD. A amostra foi constituída de 77 indivíduos de ambos os sexos, com faixa etária variando de 0 a 38 anos de idade. $\mathrm{O}$ eletrencefalograma (EEG) foi realizado em todos os pacientes. Dentre estes 20,71\% apresentaram EEG alterados, dos quais 31,3\% cursavam com epilepsia. Nos pacientes sem crises epilépticas, as alterações eletrencefalográficas foram inespecíficas, não configurando um padrão eletrencefalográfico característico da SD.
\end{abstract}

PALAVRAS-CHAVE: síndrome de Down, epilepsia, eletrencefalograma.

\section{Electroencephalografic modifications in Down syndrome}

ABSTRACT - The frequency of epilepsy in Down syndrome (DS) has been reported in literature varying from 6 to $17 \%$. A typical electroencephalografic (EEG) pattern has not been established for this condition. There is a great variation on EEG abnormalities, and most of them are not associated to behavior alterations or neurological signs. The aim of this study was to establish epidemiological and electroencephalografic parameters in institutionalised patients with clinical diagnosis of DS. We studied 77 individuals of both sexes, age ranging from 0-38 years old. The EEG was performed on all the patients; $20.7 \%$ had EEG abnormalities and $31.3 \%$ of these were epileptic. The non-epileptic patients presented inespecific EEG abnormalities. Therefore, our data did not allow us to propose a typical EEG pattern for DS.

KEY WORDS: Down syndrome, epilepsy, electrencephalogram.

A síndrome de Down (SD) é a aberração cromossômica com maior número de interpretações etiopatogênicas sobre o seu quadro clínico, sendo a cromossomopatia mais frequente, mais conhecida ${ }^{1}$ e uma das principais causas de retardo mental $(\mathrm{RM})$ de origem pré-natal ${ }^{2}$. Observações contraditórias, entretanto, têm sido feitas a respeito da frequência da epilepsia na SD, sendo esta maior que na população geral e menor que em outros grupos de $\mathrm{RM}^{3}$. Haslam ${ }^{4}$ relata uma frequência estimada de 6 a $8 \%$ nos estudos de Stafstrom et al. ${ }^{5}$. Prasher ${ }^{6}$, em uma mostra de adultos com SD, encontrou $15,9 \%$ com epilepsia, enquanto Johannsen et al. $^{7}$ referem $17 \%$ de frequência em um grupo de portadores de SD com idade entre 14 a 60 anos.

Universidade Federal da Paraíba (UFPB) e Centro Integrado de Apoio ao Portador de Deficiência FUNAD: *Neurologista da FUNAD, Mestre em Genética Médica; **Professor Doutor da Disciplina de Genética Médica. Departamento Materno-Infantil, Centro de Ciências da Saúde da UFPB; ***Médica Eletrencefalografista do Hospital Universitário da UFPB; ****Professor da Disciplina de Neurologia, CCS-UFPB, Mestre em Neuropsiquiatria. Aceite: 3-maio-1999.

Dra. Ana Moema P. Nóbrega - R. Jáder Medeiros, 101 - 58042-070 João Pessoa PB - Brasil.

E-mail: pnobrega@zaitek.com.br 
O eletrencefalograma (EEG) é um método de grande valia no diagnóstico e conduta da epilepsia. Entretanto, apesar de diversos estudos realizados, não foi possível identificar um padrão eletrencefalográfico próprio da síndrome de Down. Uma grande variação de alterações eletrencefalográficas tem sido descrita na SD, incluindo ponta (espículas), ponta-onda e complexos poli-ponta-onda. No entanto, estas anormalidades não foram bem correlacionadas com alterações comportamentais ou sinais neurológicos. Muitos indivíduos com ponta e ponta-onda frequentemente não têm antecedentes de crises, embora as alterações sejam mais frequentes quando a história é positiva. A lentificação do ritmo occipital dominante nos pacientes com SD parece estar relacionada à doença de Alzheimer, com a frequência da atividade occipital diminuindo concomitantemente com o início da deterioração cognitiva ${ }^{8-10}$. O EEG é portanto um instrumento importante no diagnóstico clínico de demência tipo Alzheimer em pacientes com $\mathrm{SD}^{8}$.

O objetivo geral deste trabalho foi identificar parâmetros epidemiológicos e eletrencefalográficos acompanhados ou não de crises epilépticas em pacientes institucionalizados com diagnóstico clínico de SD. Os objetivos específicos foram avaliar a ocorrência de alterações eletrencefalográficas e as associações com crises epilépticas; comparar as proporções encontradas de EEG alterados em pacientes com SD, com as proporções de outras populações semelhantes.

\section{MÉTODO}

A amostra foi constituída de 77 indivíduos naturais do Estado da Paraíba, com diagnóstico clínico de SD, de ambos os sexos, independente da faixa etária, em atendimento na Fundação Centro Integrado de Apoio ao Portador de Deficiência (FUNAD) e no Ambulatório de Genética Médica do Hospital Universitário Lauro Wanderley (HULW) ambos situados na cidade de João Pessoa-PB, no período de março de 1996 a junho de 1997.

Os exames eletrencefalográficos foram procedidos sem fotoestimulação em eletrencefalografo BergerED 121, 18 canais, com eletrodos de superfície distribuídos de acordo com o Sistema Internacional 10-20. Os registros foram efetuados com pacientes em vigília durante o repouso e ativados pela hiperpnéia, assim como em sonolência, sono espontâneo ou induzido por levopromazina a 4\%.

Os EEGs foram realizados nos Serviços de Eletrencefalografia do HULW-UFPB e do Posto de Atendimento Médico do Sistema Único de Saúde (SUS) - PAM Jaguaribe, na cidade de João Pessoa-PB.

No presente estudo, foi adotado o critério internacional de Pond, Bidwell e Stein apud Cockerell e Shorvon $^{11}$, o qual é baseado em evidências clínicas, considerando-se como portadores de epilepsia, os indivíduos que tenham apresentado pelo menos duas crises convulsivas nos últimos dois anos, ou que estejam em uso de anticonvulsivantes para crises anteriores a este período.

Os dados foram sistematizados, tabulados e analisados através de estatística descritiva e inferencial. Para comparação das proporções foi utilizado o teste do $\mathrm{X}^{2}$ de Pearson ${ }^{12}$.

\section{RESULTADOS}

Dos 77 portadores da SD confirmados clinicamente, 50,6\% foram do sexo masculino e 49,3\% do sexo feminino sendo a razão de sexo 1,02 (Fig 1).

A faixa etária variou de 0 a 38 anos de idade (Fig 1), sendo que a maior concentração de pacientes ocorreu entre 0 e 5 anos $(42,8 \%)$.

Dos pacientes estudados, $74 \%$ possuíam características fenotípicas predominantes do grupo racial caucasóide, $25,9 \%$ do grupo negróide. Dentre os pacientes com epilepsia, 16,7\% pertenciam ao grupo negróide.

A Tabela 1 apresenta a distribuição dos portadores de SD com e sem epilepsia por padrão eletrencefalográfico, isto é, por grafoelementos identificados no EEG.

No grupo estudado, seis indivíduos (5,4\%) foram considerados portadores de epilepsia de acordo com os critérios diagnósticos estabelecidos. Três pacientes eram do sexo masculino e três do sexo feminino, com idades variando de 4 meses a 28 anos. Destes seis pacientes, cinco apresentaram 


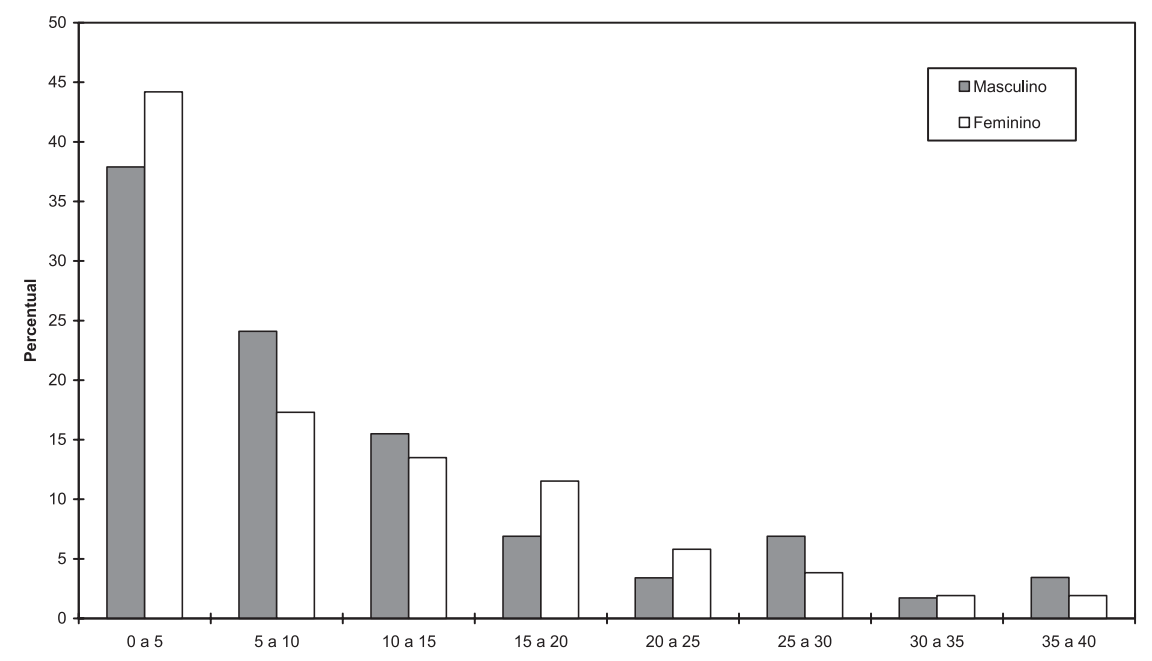

Fig 1. Distribuição dos pacientes com síndrome de Down $(n=77)$ por faixa etária e por sexo. João Pessoa - PB (1996-1997).

padrão eletrencefalográfico alterado. Todos estavam em uso de medicação anticonvulsivante por ocasião deste estudo. O início das crises epilépticas ocorreu de forma precoce no primeiro ano de vida em 50\% dos indivíduos, e dos 12 aos 25 anos nos outros três pacientes restantes. A idade média de início das crises nos seis pacientes foi 8,67 anos.

Tabela 1. Distribuição dos portadores da sindrome de Down $(n=77)$ por presença de epilepsia e por padrão eletrencefalográfico. João Pessoa - PB (1996-1997)

\begin{tabular}{|c|c|c|c|c|c|c|c|}
\hline \multirow{3}{*}{ Padrão } & \multirow{3}{*}{ Tipo } & \multicolumn{4}{|c|}{ Epilepsia } & & \\
\hline & & \multicolumn{2}{|c|}{ Presente } & \multicolumn{2}{|c|}{ Ausente } & \multicolumn{2}{|c|}{ Total } \\
\hline & & $\mathrm{n}$ & $\%$ & $\mathrm{n}$ & $\%$ & $\mathrm{n}$ & $\%$ \\
\hline Normal & & 1 & 16,66 & 60 & 84,50 & 61 & 79,22 \\
\hline \multirow[t]{2}{*}{ Lento: } & Lentificação difusa & 2 & 40,00 & 3 & 22,27 & 5 & 9,09 \\
\hline & Lentificação focal & - & - & 1 & 9,09 & 1 & 1,29 \\
\hline \multirow[t]{4}{*}{ Irritativo: } & Ponta-onda / poliponta-onda & 1 & 20,00 & 1 & 9,09 & 2 & 2,58 \\
\hline & Ponta-focal / multifocal & - & - & - & - & - & - \\
\hline & Onda aguda (Sharp) & 1 & 20,00 & 6 & 54,54 & 7 & 9,09 \\
\hline & Hipsarritmia & 1 & 20,00 & - & - & 1 & 1,29 \\
\hline Assincronia $/$ & / Assimetria & - & - & - & - & - & - \\
\hline Outros & - & - & - & - & - & - & \\
\hline Total & & 6 & 100 & 71 & 100 & 77 & 100 \\
\hline
\end{tabular}




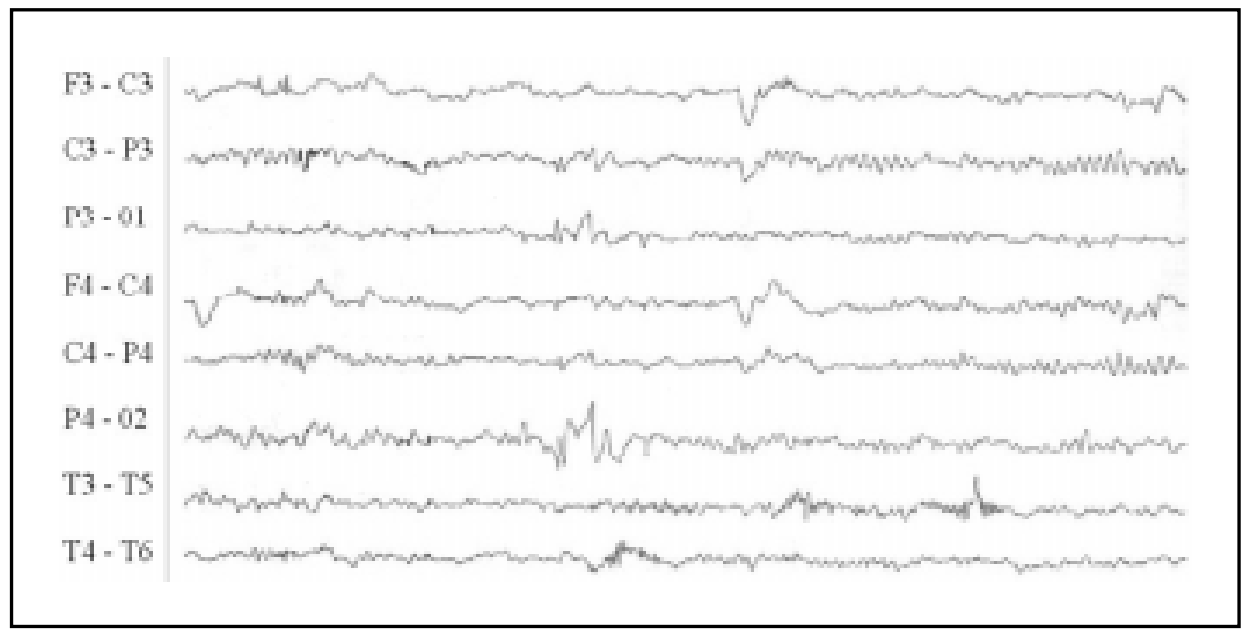

Fig 2. EEG do propósito da Família 2, em vígilia, apresentando descargas irritativas de projeção nas regiões posteriores, mais amplos a direita.

Houve predomínio das crises generalizadas $(83,3 \%)$ e, dentre estas, as crises tônico-clônicas ocorreram em $50 \%$ dos pacientes e as crises tônicas e os espasmos infantis foram diagnosticados em apenas um $(16,7 \%)$ dos indivíduos respectivamente. Um dos pacientes que apresentou espasmos infantis aos seis meses de idade evoluiu para crises tônico-clônicas generalizadas a partir dos 25 meses. As crises parciais simples com sintomas autonômicos foram detectadas também em apenas um paciente $(16,7 \%)$, a partir dos 12 anos de idade.

O EEG foi realizado em 77 portadores de SD. Nos pacientes sem crises que realizaram EEG $(\mathrm{n}=71), 11$ cursaram com alterações. Sete pacientes apresentaram descargas epileptiformes, predominando as ondas agudas em seis destes, e ponta-onda e poliponta-onda em apenas um. Atividade lenta difusa e/ou focal foi detectada em quatro pacientes.

Nos pacientes com epilepsia ( $\mathrm{n}=06$ ) o EEG apresentou alterações do padrão em cinco destes e padrão normal em apenas um (Figs 2 e 3 )

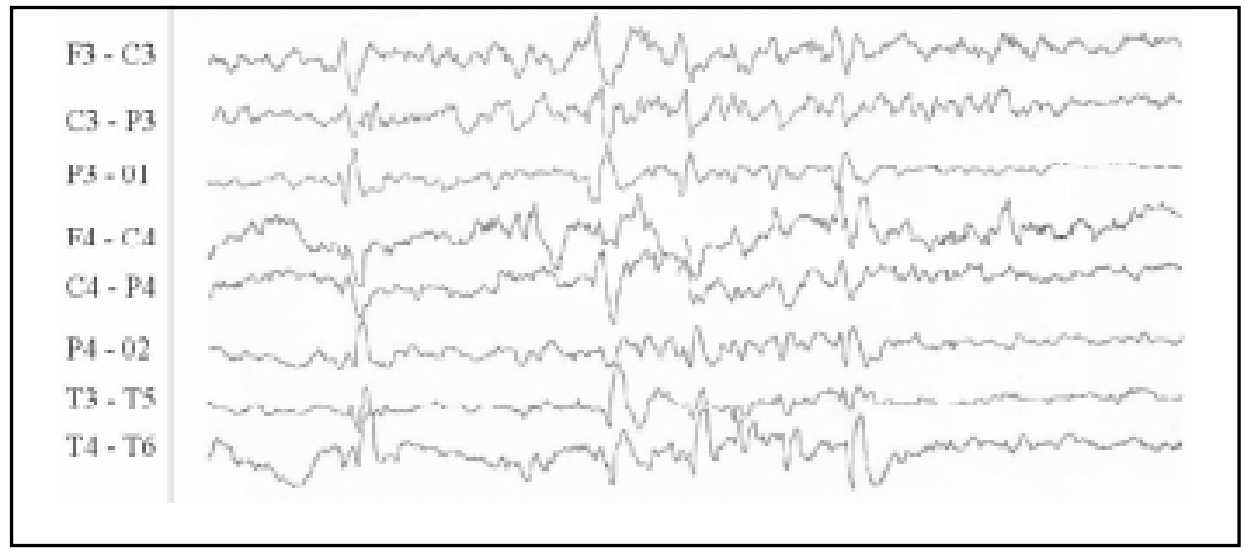

Fig 3. EEG do propósito da Família 27, em sono, com surtos iritativos bissíncronos. 


\section{DISCUSSÃO}

Guerreiro e Guerreiro ${ }^{13}$ referem que, apesar do avanço tecnológico em neuroimagem, o EEG ainda é o exame de maior sensibilidade na avaliação das epilepsias. Contudo, os trabalhos da literatura ${ }^{3,14-18}$ são concordantes em afirmarem não existir na SD, um padrão eletrencefalográfico próprio, o mesmo acontecendo com outras cromossomopatias a exemplo da síndrome do X-Frágil, que anteriormente havia sido associada a um padrão de pontas rolândicas; porém, os estudos de Guerrini et al. ${ }^{18}$ falharam em demonstrar qualquer associação significativa.

As pesquisas de Gibbs, et al ${ }^{14}$ sugerem que existem mais alterações eletrencefalográficas na $\mathrm{SD}$ do que na população geral porém menos do que em outros grupos de deficientes mentais institucionalizados. Ellingson et al. ${ }^{15}$ referem um predomínio das alterações eletrencefalográficas na infância (36 meses a 13 anos), as quais seriam variadas (pontas, ponta-onda, poliponta-onda) e geralmente não associadas às crises epilépticas, embora mais frequentes na presença destas. Ellingson, Eisen e Ottersberg apud Stafstrom et $\mathrm{al}^{19}$, relataram atividade epiléptica em $9 \%$ dos EEG analisados na sua amostra, porém apenas um paciente apresentava epilepsia clinicamente manifesta. Estes estudos demonstram que as alterações eletrencefalográficas ocorrem na SD porém não são específicas. Em vista disto, Veall ${ }^{20}$ não considerou os achados eletrencefalográficos em seus estudos de prevalência de epilepsia na SD preferindo os critérios clínicos.

Quando comparamos as proporções entre os EEG normais e os alterados (Tabela 2) do presente estudo com as mesmas proporções em diferentes populações ${ }^{14-16}$ verificamos que a diferença entre estes foi altamente significante $\left(\chi^{2}=37,74 ; p<0,001\right)$. Este resultado pode sugerir a existência de diferenças metodológicas na avaliação eletrencefalográfica desses pacientes.

Pollack et al. ${ }^{21}$, ressaltaram que nos seus cinco casos de espasmos infantis, todos apresentaram EEG com hipsarritmia, embora Coleman e Barnet apud Stafstrom et a ${ }^{19}$ referissem este padrão em apenas quatro de seus pacientes, enquanto sete seriam normais. Wolcott e Chun apud Stafstrom et a ${ }^{19}$ descreveram nos seus três casos com patologia idêntica um padrão de ponta-onda multifocal. Escofet et al. ${ }^{22}$ referiram hipsarritmia em oito dos seus nove casos de espasmos infantis, com apenas um paciente apresentando atividade paroxística multifocal com surtos intermitentes e bilaterais de ponta-onda. Silva et al. ${ }^{23}$ referiram em 13 dos seus 14 pacientes com espasmos infantis, sem lesão cerebral prévia ou cardiopatia congênita, EEG caracterizados por hipsarritmia simétrica, ausência de alterações focais (ponta ou onda lenta) após a administração de benzodiazepínicos e recorrência do padrão hipsarrítmico entre os sucessivos espasmos, características estas semelhantes às encontradas na síndrome de West idiopática.

Tabela 2. Distribuição dos pacientes institucionalizados com síndrome de Down por presença de alterações eletrencefalográficas em diferentes estudos (1964-1997).

\begin{tabular}{|c|c|c|c|c|c|}
\hline \multirow{3}{*}{ População } & \multicolumn{4}{|c|}{ EEG } & \multirow{3}{*}{ Referência } \\
\hline & \multicolumn{2}{|c|}{ Normal } & \multicolumn{2}{|c|}{ Alterado } & \\
\hline & $\mathrm{n} / \mathrm{N}$ & $\%$ & $\mathrm{n} / \mathrm{N}$ & $\%$ & \\
\hline Illinois / EUA & $119 / 184$ & 64,67 & $65 / 184$ & 35,32 & Bibbs et al. (1964) \\
\hline Nebrasca / EUA & $72 / 94$ & 76,59 & $22 / 94$ & 23,40 & Ellingson et al. (1970) \\
\hline Lancaster / Inglaterra & $54 / 120$ & 45,00 & $66 / 120$ & 55,00 & Tangye (1979) \\
\hline João Pessoa-PB / Brasil & $61 / 77$ & 79,22 & $16 / 77$ & 20,77 & Presente estudo \\
\hline Total & $306 / 475$ & 64,00 & $169 / 475$ & 36,00 & \\
\hline
\end{tabular}


Tangye $^{16}$ encontrou EEG normais em $42,2 \%$ dos seus pacientes. Dentre os alterados foi observado predomínio de ondas "teta", sugerindo imaturidade. Nos casos de início tardio, após os 43 anos, quatro traçados apresentavam ondas agudas e outros quatro atividade irritativa paroxística. Guerrini et al. ${ }^{18}$ não conseguiram estabelecer também um padrão eletroclínico para as crises reflexas em seis dos seus pacientes com SD, encontrando os mais diversos traçados eletrencefalográficos, demonstrando a heterogeneidade dos padrões eletroclínicos na epilepsia reflexa.

Um longo espectro de alterações eletrencefalográficas também foi referido por Stafstrom et al. ${ }^{5}$ Os EEG dos pacientes com crises de etiologia conhecida, geralmente refletiam a doença de base, de forma que lesões que levam a crises parciais mostraram pontas ou lentificação focais. Entretanto em muitos casos, lentificação generalizada ou combinação de pontas e lentificação não são preditivos de uma etiologia específica. Nos pacientes que apresentavam crises de etiologia desconhecida, a lentificação generalizada foi a alteração mais frequentemente encontrada. Os referidos autores encontraram grande especificidade nos casos de espasmos infantis pela presença em todos os pacientes do padrão tipo hipsarritmia, porém concluíram que os achados eletroclínicos não foram sufícientes para distinguir as crises de etiologia conhecida daquelas de etiologia desconhecida. Entretanto, tais achados foram úteis na classificação e seguimento clínico das mesmas.

Kaneko et al..$^{25}$ em estudo comparativo de achados eletrencefalográficos de portadores de SD e portadores da síndrome feto-alcoólica (SFA) encontraram, nas crianças com SD, lentificação difusa do EEG, enquanto nas crianças com SFA havia diminuição da atividade, particularmente na frequência alfa ena ausência de atividade lenta significativa. Nas crianças com SD uma diminuição significativa de atividade alfa foi encontrada nas regiões corticais posteriores, enquanto na SFA as crianças eram mais afetadas no hemisfério esquerdo, o que poderia servir para caracterização neurofisiológica de SFA.

Johannsen et al. ${ }^{7}$ observaram que no EEG realizado em 50 dos 85 portadores de SD, dos 12 indivíduos com epilepsia dois tinham EEG normal e 13 dos pacientes sem crises tinham EEG alterado.

De acordo com Adams, Victor e Ropper ${ }^{26}$, muitos pacientes epilépticos apresentaram EEG interictal normal, enquanto pequeno número de pessoas saudáveis (2 a $3 \%$ ) mostram alterações paroxísticas no EEG, sendo que algumas delas têm história familiar de epilepsia e podem desenvolver crises posteriormente. Na presente casuística, entre os 11 pacientes não epilépticos com EEG alterado, três $(27,2 \%)$ tinham história familiar de epilepsia.

As alterações eletrencefalográficas encontradas nos seis pacientes com epilepsia foram predominantemente do tipo irritativo em três (ponta-onda, poliponta-onda, onda aguda, hipsarritmia), e apenas dois dos pacientes apresentaram padrão de lentificação difusa (ondas "teta" e delta). Estes achados foram comparados com o grupo com SD porém sem epilepsia, nos quais a atividade irritativa foi observada em sete $(63,6 \%)$ e atividade lenta difusa ou focal em três $(27,2 \%)$ dos casos. Comparando ainda os dois grupos por faixa etária, encontramos em ambos maior proporção de EEG alterado (11,7\%), em pacientes abaixo dos 15 anos. Apenas três pacientes estavam acima desta idade predominando neles uma lentificação difusa.

Os resultados do presente estudo quanto à faixa etária e variabilidade dos grafoelementos estão de acordo com os de Ellingson et al. ${ }^{15}$ que encontraram um predomínio das alterações eletrencefalográficas na infância, com padrão bastante variado. Pueschel et al. ${ }^{27}$ referem em uma "cohort" de 450 indivíduos com SD, 40\% dos pacientes iniciando as crises epilépticas também antes do primeiro ano de vida e outros $40 \%$ na terceira década. Concordam estes resultados também com os dados apresentados por Gibbs et al. ${ }^{14}$ quanto à raridade das espículas positivas $14 \mathrm{a} 6 \mathrm{~Hz} / \mathrm{s} \mathrm{na}$ população com SD, não tendo sido encontrado nenhum paciente na amostra com este padrão eletrencefalográfico, o qual de acordo com os referidos autores está presente em $20 \%$ da população de escolares normais, sendo aceito atualmente como uma variável benigna e inespecífica no EEG. 
Os principais resultados do presente estudo são:

1. A prevalência estimada da epilepsia em portadores da síndrome de Down foi 54/1000.

2. A idade média de início da epilepsia foi 8 anos, sendo que $83,3 \%$ dos casos iniciaram em pacientes com idade igual ou menor que 15 anos.

3. Todos os pacientes da amostra realizaram EEG; destes 20,7\% apresentaram EEG alterado, dos quais $31,3 \%$ cursavam com epilepsia.

4. Crises generalizadas do tipo tônico-clônicas ocorreram em $50 \%$ dos casos.

Estes resultados reforçam a concepção atual de indefinição do padrão eletrencefalográfico na $\mathrm{SD}$, além de constatar um percentual elevado de EEG alterado nestes pacientes, tanto nos que cursavam com epilepsia $(83,3 \%)$ quanto naqueles sem epilepsia (15\%).

As crianças portadoras de SD e epilepsia devem ser avaliadas com critérios bem definidos para se determinar se existe alguma associação tal como com acidente vascular cerebral ou infecção, entre outras.

Agradecimentos - Os autores agradecem ao Professor José Correia de Farias Brito, da Disciplina de Neurologia do Departamento de Medicina Interna, CCS-UFPB, pela leitura crítica do artigo e pelas sugestões apresentadas.

\section{REFERÊNCIAS}

1. Penchaszadeh VB. Genetics and public health. Bull Pan Am Health Organ 1994;28:62-72.

2. Oteiza MEF, Dorticós AP, Iglesia DG. Estudio de la incidencia del sindrome de Down em Cuba. Ver Cuba Pediatr 1992;64:148-153.

3. McVicker RW, Shanks OEP, McClelland RJ. Prevalence and associated features of epilepsy in adults with Down's syndrome. Br J Psychiatry 1994;164:528-532.

4. Haslam RHA. Neurological problems associated with Down syndrome. In Nadel L (ed.). Down syndrome: living and learning in the community. New York: Wiley-Liss, 1995.

5. Stafstrom CE. Epilepsy in Down syndroime: clinical aspects and possible mechanism. Am J Men Ret 1993;98(Suppl):12-26.

6. Prascher VP. Epilepsy and associated effects on adaptative behaviour in adults with Down syndrome. Seizure 1995;4 53:56.

7. Johannsen P. Christensen JE, Goldstein H, Nielsen VK, Mai J. Epilepsy in Down syndrome prevalence in three age groups. Seizure 1996;5:121-125.

8. Visser FE, Kuilmam M, Oosting J, van Ruffelen AC. Use of eletroencephalography to detect Alzheimer's disease in Down's syndrome. Acta Neurol Scand 1996;94:97-103.

9. PolitoffAL, Stadter RP, Monson N, Hass P. Cognition-related EEG abnormalities in non-demented Down syndrome subjects. Dementia 1996;7:69-75.

10. Partanem J, Soininen H, Knnen M, Kilpelinen R, Helkala EL, Riekkinen P. EEG reactivity correlates with neuropsychological test scores in Down syndrome. Acta Neurol Scand 1996;94:242-246.

11. Cockerell CCO, Shorvon SD. Epilepsia: conceitos atuais. São Paulo: Lemos Editorial, 1997.

12. Daniel WW. Biostatistcs: a foundation for analysis in the health ciences. New York: John Wiley, 1976.

13. Guerreiro CAM, Guerreiro MM. Epilepsia. 2.Ed. São Paulo: Lemos Editorial, 1996.

14. Gibbs EL, Gibbs FA, Hirsch W. Rarity of 14 - and 6 - per - second positive spiking among mongoloids. Neurology 1964;14:581-583.

15. Ellingson RJ, Menolascino FJ, Eisen JD. Clinical-EEG relationships in mongoloid confirmed by karyotipe. Am J Ment Defic Res 1970;74:645-650.

16. Tangye SR. The EEG and incidence of epilepsy in Down's syndrome. J Ment Defic Res 1979;23:17-24.

17. Guerrine R, Dravet C, Ferrari AR, et al. Evoluzione dell' epilessia nel piú frequenti forme genetiche com ritardo mentale (sindrome de Down e sindrome dell' X fragile). Pediatr Med Chir 1993;15:19-22.

18. Li LM, O’Donoghue MF, Sander JWAS. Myoclonic epilepsy of late onset in trissomy 21. Arq Neuropsiquiatr 1995;53:792-794.

19. Stafstrom CE, Patxot OF, Gilmore HE, Wisniewski KE. Seizures in childen with Down syndrome: etiology, characteristics and outcome. Dev Med Child Neurol 1991;33:191-200.

20. Veall RM. The prevalence of epilepsy among mongols related to age. J Ment Defic Res 1974;18:99-106.

21. Pollack, MA, Golden GS, Schmidt R, et al. Infantile spasms in Down syndrome: a report of 5 cases and review of the literature. Ann Neurol 1978;3:406-408.

22. Escofet C, Valbuena O, Gassie R, Sanmart FX, Campistol J. Espasmos infantiles in niños con sindrome de Down / infantile spasms in children with Down's syndrome. Ver Neurol 1995;23:315-315.

23. Silva ML, Cieuta C, Guerrine R, Plouin P, Livet MO, Dulac O Early clinical and EEG features of infantile spasms in Down syndrome. Epilepsia 1996;37:977-982.

24. Guerrini R, Genton P, Bureau M, Dravet C, Roger J. Reflex seizures are frequent in patients with Down syndrome and Epilepsy. Epilepsia 1990;31:406-417.

25. Kaneko WM, Phillips EL, Riley EP, Ehlers CL. EEG findings in fetal alcohol syndrome and Down syndrome children. Electroencephalogr Clin Neurophysiol 1996;98:20-28.

26. Adams RD, Victor M, Ropper AH. Principles of neurology. New York: McGraw-Hill, 1997.

27. Pueschel SM, Louis S, McKnight P. Seizure disorders in Down syndrome. Arch Neurol 1991;48:318-320. 